\title{
Technological design processes of vibration processing of particularly accurate parts of agricultural machinery
}

\author{
Mikhail Tamarkin ${ }^{1}$,Elina Tishchenko ${ }^{1, *}$, and Elena Murugova ${ }^{1}$ \\ ${ }^{1}$ Don State Technical University, 1, Gagarin sq., Rostov-on-Don, 344003, Russia
}

\begin{abstract}
This paper presents the research results on the process of vibration abrasive processing of particularly accurate parts of agricultural machinery. Process peculiarities are described. The theoretical studies of metal removal calculation, surface roughness and processing time for solving various technological problems are presented. Experimental studies have been carried out confirming the adequacy of theoretical formulas. Process design procedure has been developed.
\end{abstract}

\section{Introduction}

Vibration processing in abrasive medium (VP) is one of the processing methods with free abrasives. The wide application of this method in the operations of workpiece cleaning, removing burrs, rounding sharp edges, coatings, polishing, finishing the surface of agricultural machinery parts in order to improve its quality is repeatedly discussed, though not enough disseminated, especially the possibility of equipment acquisition and working media [1-15].

The main advantage of free abrasive processing is a more complete application of the grains cutting ability than when processed with a fixed abrasive, as their cutting edges are evenly distributed on the surfaces, as well as reoriented and moved during processing. VP has enough wide technological capabilities and allows carrying out a high-performance processing with relatively simple kinematic circuits of equipment. Compared to the fixed abrasive processing methods, the temperature mode of the process is significantly reduced, since the cutting speeds are lower than, for example, in conventional grinding, and the micro-cutting zones are abundantly flowed round with the process liquid. This makes it possible to obtain high quality of the processed surfaces without burns, micro cracks and undesirable structural changes. Agricultural machine parts made of various materials, having different resistance to abrasive destruction, both simple and complex, the dimensions of which are in the range from several millimeters to several meters, and parts of low stiffness, parts with internal cavities, are subjected to processing.

The processing of the precise parts of agricultural machines has a widespread appeal. The purpose of any processing is to produce parts with a specified accuracy. In its turn, accuracy is the degree to which the result of measurement, calculation or specification

\footnotetext{
* Corresponding author: lina_tishenko@mail.ru
} 
conforms to the geometrically correct prototype, i.e. compliance of the dimensions and shape of the processed surfaces with the specified accuracy, as well as mutual arrangement of individual sections, part surface finish in compliance with the drawing requirements and specifications.

The accuracy and quality of the processed parts surfaces are subjected to high requirements, since the durability of the machines depends mainly on the quality and purity of the parts surfaces. Despite the high accuracy of modern metal cutting equipment, it is impossible to obtain accurate dimensions in accordance with the fixed drawing requirements. All processed parts will have some deviations in one side or the other. At the same time, it is the finishing operations that determine the accuracy of the dimensions and the quality of the parts surface, so the research in the field of finishing processing methods, including free abrasive medium, is especially relevant.

During vibration abrasive processing, the parts are loaded into the working chamber filled with the working medium of the required characteristic (Fig. 1). The working chamber mounted on the spring supports can oscillate in different directions [1].

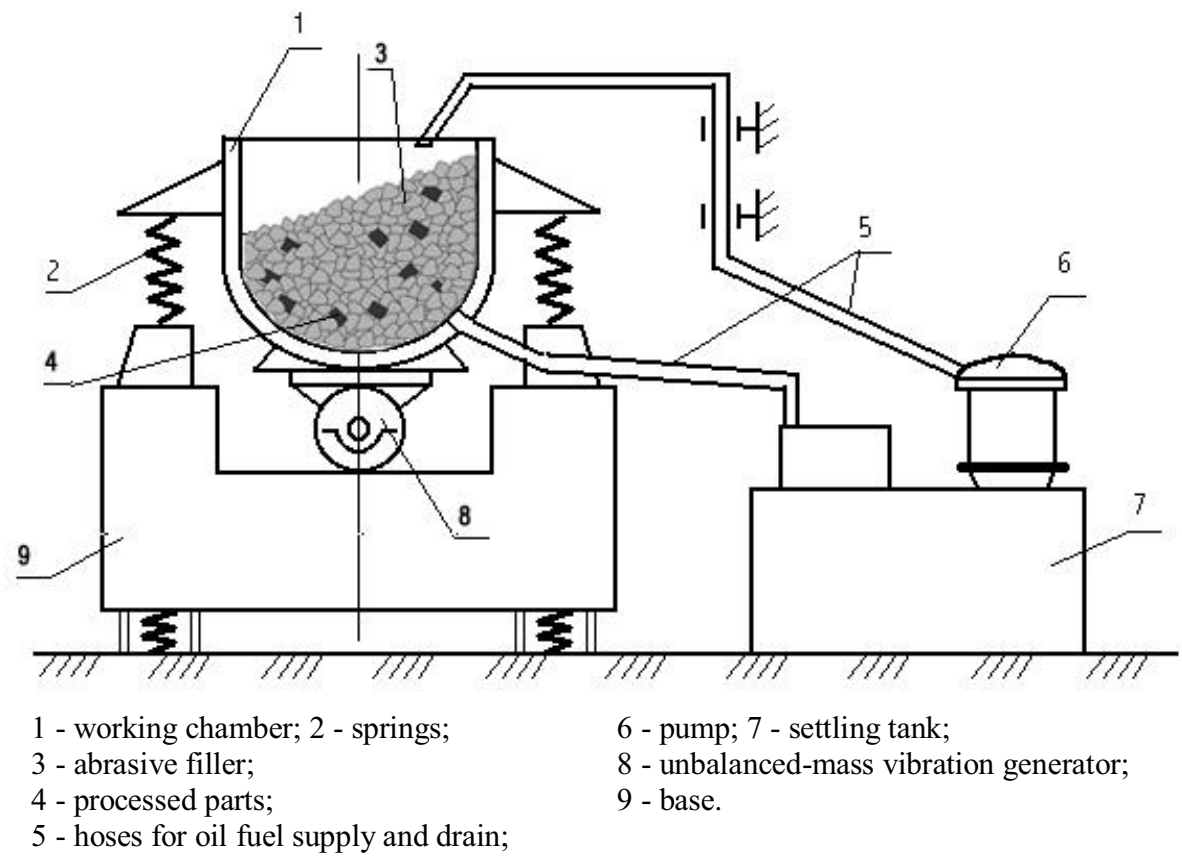

Fig. 1. Vibration processing machine diagram.

The working chamber receives the drive from an inertial vibrator (a rotating shaft with unbalanced weights) with a frequency of $15-50 \mathrm{~Hz}$ and an amplitude of 0.5 to $6-8 \mathrm{~mm}$, during the vibration process the processed parts and particles of the working medium move relatively, performing two types of movements: oscillation and slow rotation of the entire mass (circulation movement). From the walls of the working chamber, vibration is transmitted to adjacent layers of the working medium, which transfer it to the following layers, etc. During processing, the parts occupy different positions in the working environment, which ensures a uniform treatment of all surfaces contacting the working medium. The large number of micro shocks acting on the workpiece simultaneously in different directions contributes to some extent to keeping it in a suspended state, thus eliminating coarse nicks and damage. Because of circulation, processing takes place in all 
zones of the working chamber. The most efficient processing is near the bottom of the chamber, where the pressure of the working medium is higher.

The processing is carried out with continuous or periodic supply of process liquid to the working chamber, which ensures removal of wear products (metal and abrasive particles) from the parts and particles surface of the working medium, wetting parts and medium, helps their separation and uniform distribution of parts in the working medium. By changing the level of liquid in the working chamber, the processing intensity can be controlled. Various kinds of chemical additives with the special properties can be added to the oil fuel composition, which also allows controlling the intensity of the process and the quality of treatment. The liquid facilitates workpieces cooling.

The vibration processing intensity depends on the modes and duration of processing; characteristics and particle sizes of the working medium; the working chamber volume and the degree of its filling; volume ratio of the processed parts and abrasive granules; mechanical properties of the processed parts material and their mass, etc. The most significant factors are the graininess of the abrasive granules and the contact forces of the surfaces with the working medium.

The main parameters that characterize this process are the nature of the movement (trajectory) of the working chamber and the particles of the working medium, their speed and acceleration, the forces of micro shocks, contact pressures, stresses and temperatures arising in the area of micro shocks, the nature of the part material destruction.

During processing, the working chamber makes harmonic or close to them oscillations, and its points move along trajectories in the form of a circle or ellipse.

\section{Materials and Methods}

Ellipsoid parameters of the medium particle contact with the part surface are determined during theoretical studies of processing. The major and minor axis of the ellipsoid can be determined by the following formulas [1]:

$$
\begin{gathered}
b=\sqrt{R^{2}-\left(R-h_{\max }\right)^{2}} \\
a=\frac{\pi}{2}(\operatorname{ctg} \beta-f) h_{\max }+b
\end{gathered}
$$

where $\mathrm{R}$ - the particle radius; $\beta$ - the impact angle of the abrasive particle; $f$ - coefficient of abrasive grain friction over metal; $h_{\max }$ - maximum depth of medium particle penetration into the part surface

To determine the maximum depth of particle penetration the following dependence is obtained:

$$
h_{\max }=2 V_{e f} R \sin \beta \sqrt{\frac{\rho_{r}}{3 k_{R} c \sigma_{s}}}
$$

where $V_{e f}$ - the effective velocity of the abrasive particle; $k_{R}$ - coefficient that takes into account the effect of abrasive particle grain on the actual contact area; $\rho_{r}$ - density of the particle material; c - coefficient that evaluates the bearing capacity of the contact surface; $\sigma_{S}$ - the yield strength of the part material. 
The dependence for the determination of metal removal in one impact of abrasive particle is obtained:

$$
q=15.5 k_{c} k_{z} R^{3}\left(V_{e f} \sin \beta \sqrt{\frac{\rho_{r}}{3 k_{R} c \sigma_{s}}}\right)^{5 / 2}(\operatorname{ctg} \beta-f) \rho_{d}
$$

$k_{c}$-the chip formation coefficient; $k_{z}$ - coefficient that takes into account the effect of abrasive particle grain on metal removal; $\rho_{d}$ - the part material density.

Based on the carried studies, the removal of metal from the entire part surface will be determined by the following formulas:

$$
\begin{aligned}
& Q=P_{1} P_{2} \omega t q \frac{S_{\mathrm{det}}}{4 R^{2}} \quad \text { at } S_{\mathrm{det}} \succ 4 R^{2} \\
& Q^{\prime}=P_{1}^{\prime} P_{2} \omega t q \text { at } S_{\mathrm{det}} \prec 4 R^{2}
\end{aligned}
$$

where $S_{\mathrm{det}}$ - the surface area of the part; $P_{1}\left(P_{1}^{\prime}\right)$ - the geometric probability of the event that any point of the package square is covered with a contact spot in one action cycle of the abrasive particles mass; $P_{2}$. the probability of the event that the interaction of the abrasive particle with the part surface will lead to micro-cutting; $t$ _ is the processing time, $\omega$ - is the oscillation frequency of the working chamber.

When processing precise parts, the amount of layer removed from the part is of great importance. We define it by dependence:

$$
h=\frac{Q}{4 R^{2} \rho_{d}}=\frac{P_{1} P_{2} \omega t q}{4 R^{2} \rho_{d}}
$$

When examining the mechanism of the roughness formation of the treated surface, a dependence is obtained for calculating the average arithmetic deviation of the roughness profile at vibration processing:

$$
R a=0.09 \sqrt{\frac{h_{\max } L_{e d}}{z_{0}}}
$$

where $z_{0}$ - the number of grains above the surface unit of the bundle, $L_{e d}$ - the unit length.

The formation of surface roughness during processing in a free abrasive medium has the following features: after completely removing the profile of the initial roughness and while continuing the processing, the so-called steady roughness is formed on the part surface, which is reproduced over time.

Determination of processing time is one of the most important tasks of mechanical engineering technology, without which it is impossible to design technological processes. Dependencies to determine the following time are obtained:

- achievement of steady roughness 


$$
t_{R a}=\frac{4 k_{p r} R z_{i} R^{2}}{P_{1} P_{2} f_{b} V}
$$

where $k_{p r}-$ a coefficient of the profile view of the initial roughness; $R z_{i}$ - the initial roughness of the surface; $f_{b}$ - the cycle frequency of the abrasive particle mass on the part surface; $V$ - the metal volume removed in one stroke of the abrasive particle. It should be noted that the time to achieve the steady-state roughness is directly proportional to the height of the initial roughness.

- Removal of burrs and debris

During the initial processing period, the burr is either wrapped at the base or deformed and pressed against the part surface. Then it is clipped at the base, the main part of the burr falls off, and the base is gradually removed with further rounding of the sharp edge. We accept provisionally that on average the height of such a burr is equal to its thickness. In view of the above, the following formula is proposed for calculating the time required to remove burrs and debris:

$$
t_{z}=k_{z a u} \frac{4 L_{z a u}^{3} R^{2}}{\pi a b P_{2} f_{b} V} .
$$

where $k_{z a u}$ - a coefficient that takes into account the special location of the burr on the part, $L_{z a u}$ - the thickness of the burr at the base. The resulting model allows calculating the time removal of burrs and debris; moreover, that time is directly proportional to the thickness of the burrs at the base.

- Round sharp edges

Round let the sharp edge on the part with a radius $r$.

$$
t_{r}=k_{r} \frac{0.85 R^{2} r^{3}}{\pi a b P_{2} f_{b} V} .
$$

where $k_{r}$ - A coefficient that takes into account the specific conditions of the abrasive particle interaction with the edge of the part.

Experimental studies of the vibration processing of precise parts were carried out. For the experiments, the samples from various materials (steel, aluminum alloys, and brass) were used, made in terms of six, seven and eight qualities of accuracy. To maintain the part accuracy specified by the designer, it is necessary that the actual sample size obtained after vibration processing have a value in the specified tolerance field. Therefore, it is necessary to know the difference between the actual size and the limiting size of the sample before performing vibration processing. The value of the actual sample size is close to the smallest limit size. In this regard, incoming control is required. For the samples that have a valid size close to the smallest limit size, adjust the treatment time downward or leave the vibration processing allowance within the metal removal range during the processing time.

When conducting experiments to study removal from the surface of the sample, the four of the sample from each test material were processed. The following abrasive media were used: one - abrasive white-green cone on a polymer binder with a grain of eight, 2 abrasive yellow cone with the grain of 15,3 - trihedral white prisms PT $15 \times 15$ grain of 25 . Processing modes: chamber oscillation frequency $-26 \mathrm{~Hz}$, oscillation amplitude $-1.5-2 \mathrm{~mm}$. 
After every 10 minutes, the samples were removed from the chamber and weighed. Experimentally, the height of the removed layer was determined by the formula:

$$
h=\frac{m}{S_{\mathrm{det}} \rho_{d}}
$$

where $\mathrm{m}$ - mass of the removed metal.

Figure 1-3 compares the results of theoretical and experimental studies. Solid lines show the lines calculated by theoretical constraints. Red circles show the results of experimental studies in abrasive medium with grain 8 , blue squares - the results of experimental studies in medium with grain 15 , green ovals - the results of experimental studies in medium with grain 25 . Confidence intervals with a confidence probability of $95 \%$ are constructed.

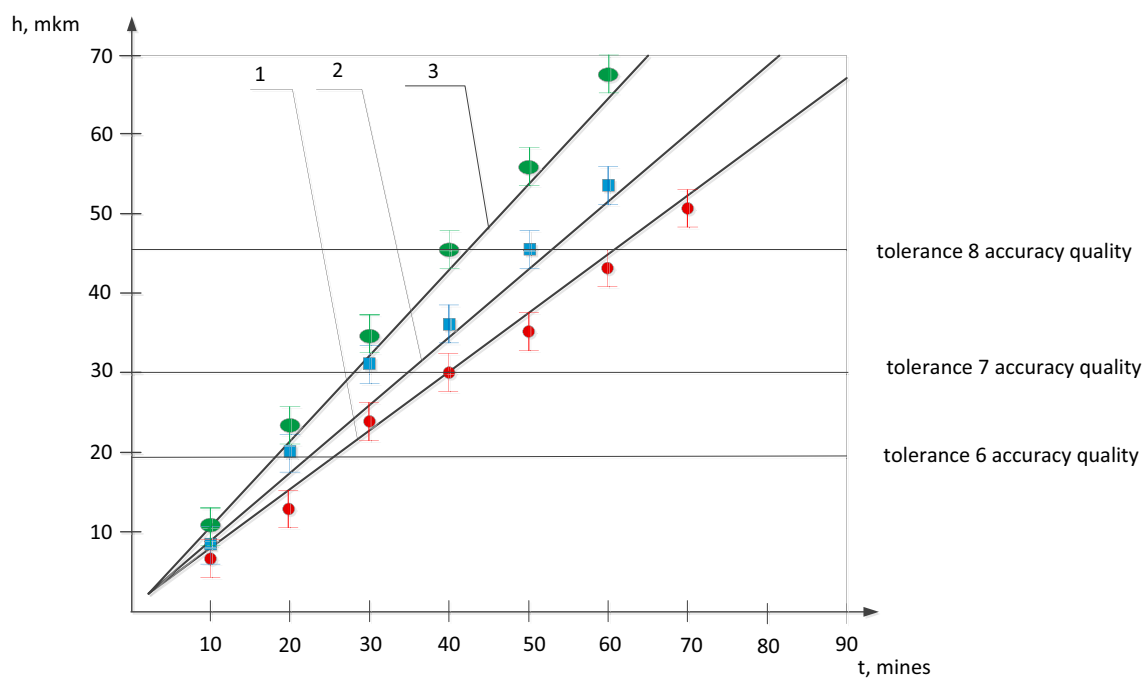

Fig. 1. The dependance of the height of the removed layer on the processing time. The part material steel 45 . 


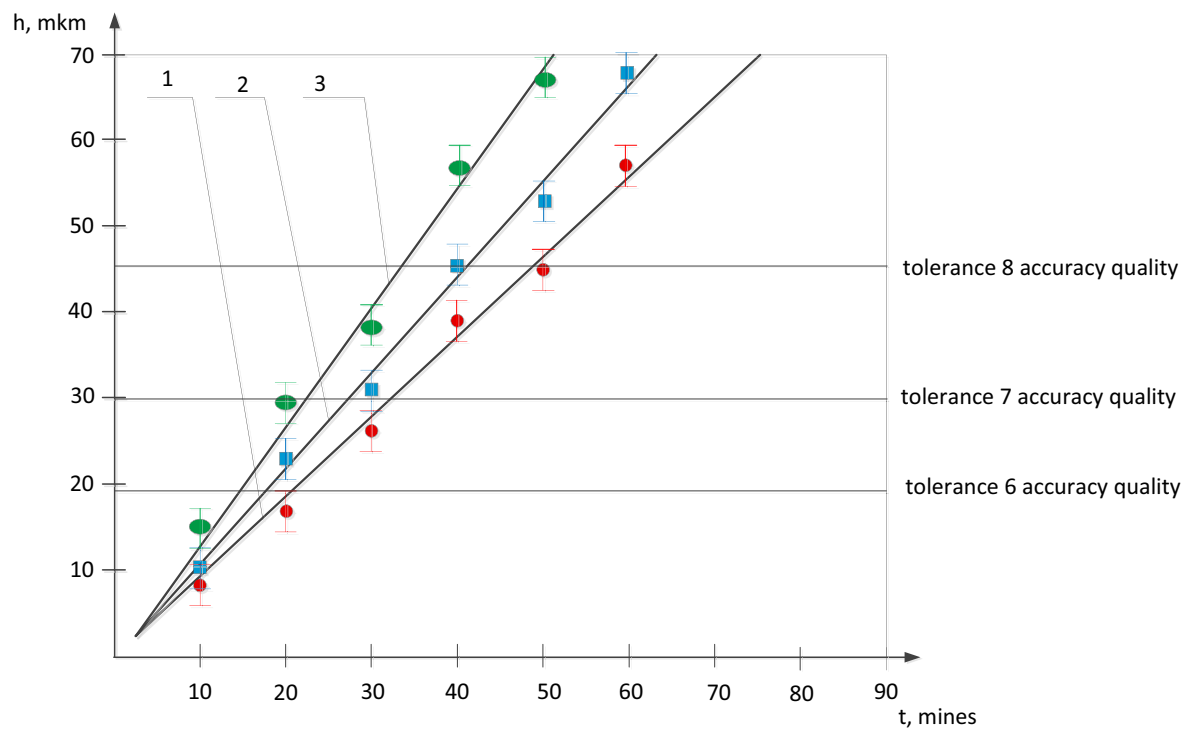

Fig. 2. The dependance of the height of the removed layer on the processing time. The part material is B95.

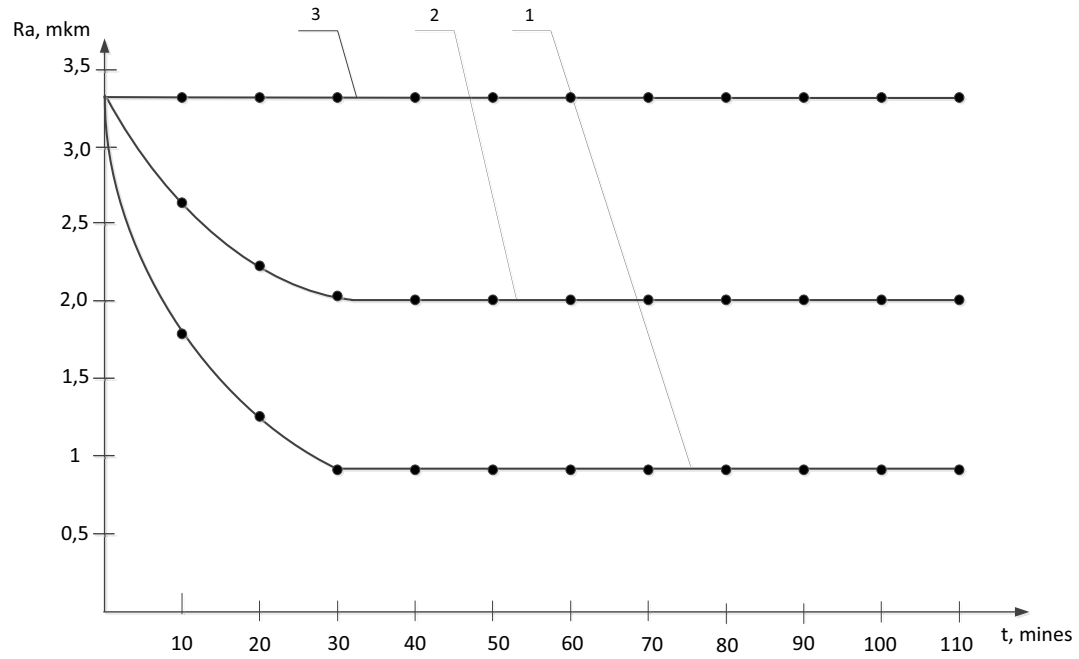

Fig. 3. Dependence of the height of the removed layer on the processing time. The material of the part is brass LS59-1.

Based on the study results, the following conclusions can be drawn:

1. When conducting studies, there is a high convergence of the theoretical results and experimental data. The discrepancy does not exceed $20 \%$, which indicates the adequacy of the results obtained in experimental studies.

2. Vibration processing in abrasive granular media enables to process the high precision parts. At the same time, for the steel samples with high yield strength (600 MPa or more) after 40-70 minutes of processing, accuracy is ensured within the established tolerance, for samples from non-ferrous alloys with lower yield strength, this time is reduced to 15-30 minutes. 
3. With the abrasive medium graininess increases, the processing time decreases. In this case, for high-precision samples from non-ferrous alloys, an abrasive medium with a low grain content is recommended.

4. As the vibration amplitude of the chamber increases, the processing time decreases. It is recommended to process samples of high accuracy of six and seven squares with amplitude not exceeding $2 \mathrm{~mm}$.

5. To maintain the part accuracy specified by the designer, it is necessary to leave the allowance for vibration processing within the limits of metal removal during processing in accordance with the assigned modes.

When examining the surface roughness of samples from various materials, the same treatment modes and abrasive media were used as in the removal study. The purpose of the experiments was to establish the influence of technological modes and the characteristics of working media on the value of a steady roughness of high-precision parts made according to 6-8 accuracy standards. The initial roughness of the samples of 8 square meters was Ra $3.2 \mathrm{mkm}, 6$ and 7 square meters - Ra $2.5 \mathrm{mkm}$.

Figure 4-5 shows the curves describing the surface roughness of the sample.

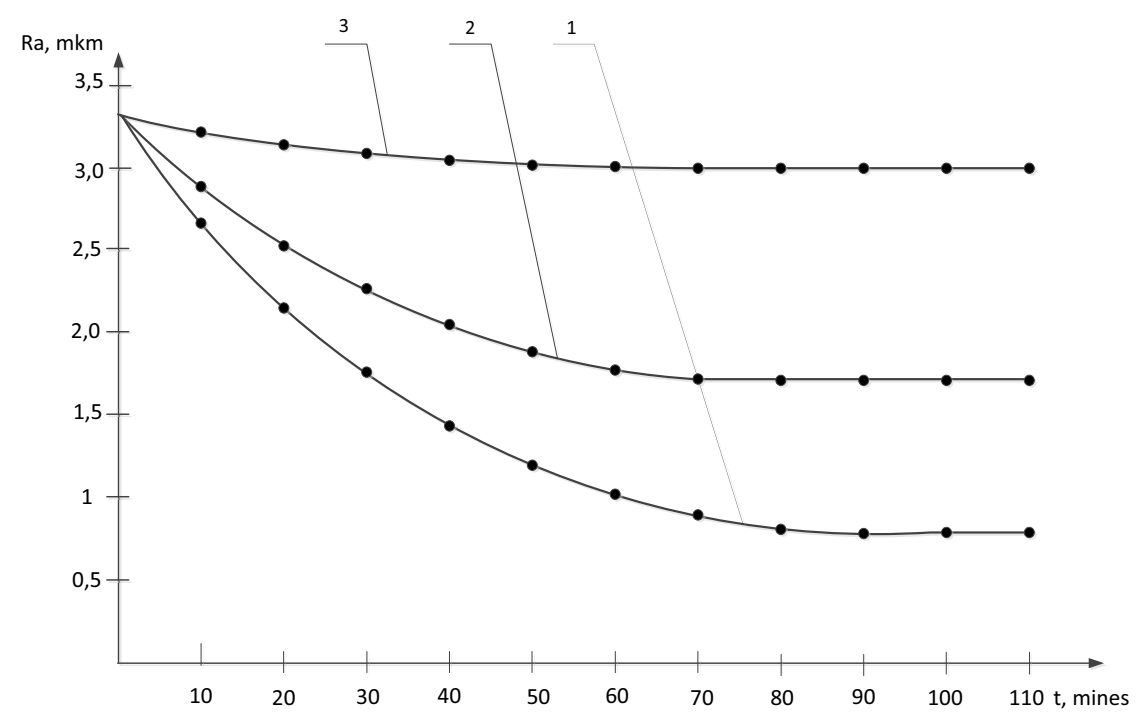

Fig. 4. The dependence of the surface roughness on the processing time. Part material - steel 45. Accuracy degree of details -8 . 


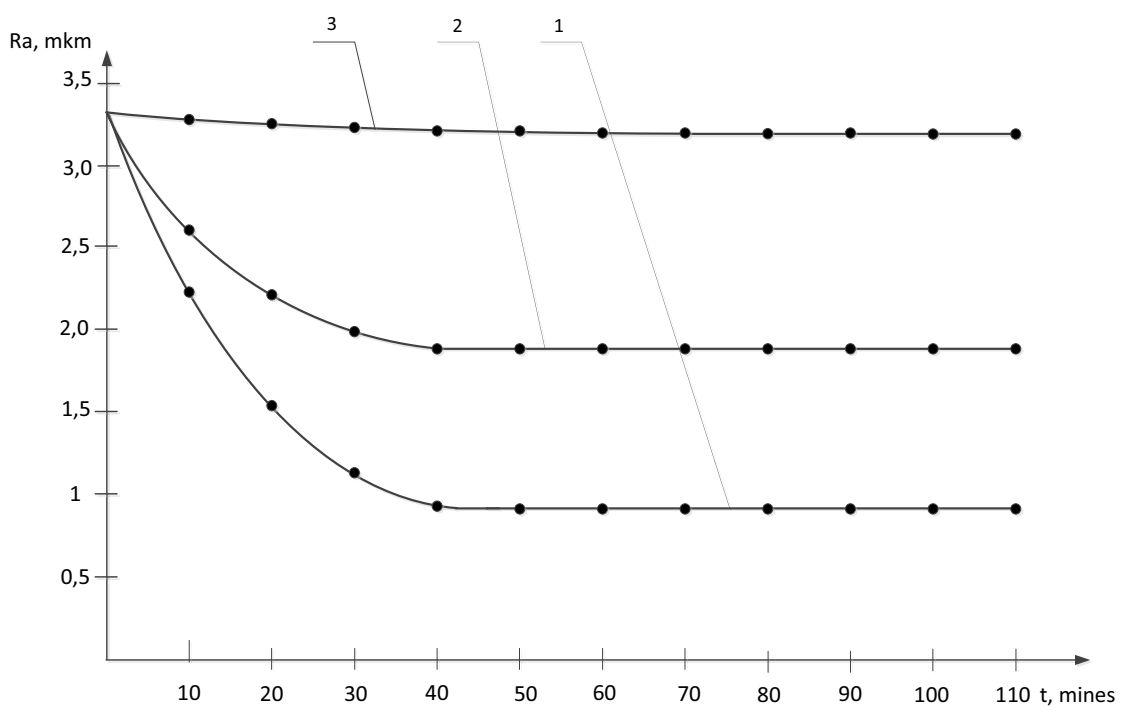

Fig. 5. The dependence of surface roughness on the processing time. The part material is B95. Accuracy degree of details -8 .

During studies on the surface of the sample, a steady roughness is formed in accordance with Table 1.

Table 1. Average arithmetic deviation of the steady-state roughness profile $\mathrm{Ra}(\mu \mathrm{m})$ at vibration processing.

\begin{tabular}{|c|c|c|c|}
\hline \multirow{3}{*}{$\begin{array}{l}\text { Abrasive grain } \\
\text { size as per } \\
\text { GOST 3647-71 }\end{array}$} & \multicolumn{3}{|c|}{ Sample material } \\
\hline & Steel 45 & B95 & Brass LS 59-1 \\
\hline & $\left(\sigma_{S}=600 \mathrm{mPa}\right)$ & $\left(\sigma_{S}=400 \mathrm{mPa}\right)$ & $\left(\sigma_{S}=\mathbf{3 0 0} \mathrm{mPa}\right)$ \\
\hline 25 & 2.9 & 3.1 & 3.2 \\
\hline 16 & 1.7 & 1.9 & 2.0 \\
\hline 8 & 0.8 & 0.9 & 0.9 \\
\hline
\end{tabular}

Based on the study results, the following conclusions can be drawn:

1. The curves obtained in the graphs confirm the surface roughness formation described in Chapter 2 during vibration processing in a free abrasive medium and are exponential in nature.

2. A steady surface roughness is observed.

3. The values of steady surface roughness for samples from all analyzed materials in abrasive medium of different grain size are established. With an increase in abrasive medium grain, the steady surface roughness of non-ferrous alloy samples exceeds the initial one, which indicates the inadmissibility of its use for processing high-precision parts.

\section{Results}

To ensure the best results of processing of machine parts with the high accuracy at the lowest cost, it is necessary to develop a methodology for choosing rational process parameters. Process indicators such as high productivity or low cost can be used as rationality criteria. Moreover, the part must not lose its specified accuracy. 
When defining the values of controlled process factors, it is advisable to minimize their amount by limiting the most significant ones, which allow managing effectively the results of the process.

Thus, the input parameters are as follows:

- Initial and specified value of surface roughness Ra,

- Specified removal of metal Q (height of removed layer $h$ ),

- Characteristics of the part material: yield strength, Brinell hardness of the material, coefficient of bearing capacity of the contact surface,

- Dimensions and graininess of abrasive medium particles,

- Amplitude and oscillation frequency of the chamber,

- Machine model,

The results of vibration processing can be reviewed by the following criteria: surface layer roughness, metal removal from the surface, required radius of sharp edge rounding.

The minimum cost and processing labor can be used as the indicators of objective economic function. At the same time, the quality indicators of the surface layer or the necessary removal can be used as limiting functions.

When performing calculations, those options that do not satisfy restrictive functions are discarded. From the remaining options, the variant that provides a minimum of the objective function is selected. This variant is accepted for the production realization.

Based on the results of theoretical and experimental studies, the methodology for calculating rational processing modes can be proposed.

Methodology of rational modes calculation:

1. Depending on the size and configuration of the part, it is necessary to determine the size of the abrasive particles and select the abrasive material.

2. Depending on the part material and the required precision, roughness or other processing parameter (for example, the radius of the sharp edge round), we select several combinations of machining modes using an abrasive of different grain.

By the formulas, we determine theoretical values of the metal removal from the part surface, steady roughness or radius of edge rounding depending on the required tasks, and select processing modes and abrasive medium. We leave only those modes that correspond to the set tasks.

From the above formulas, we determine the processing time to obtain the specified processing parameters. From the previously selected modes, we select that one where the processing is carried out in less time. Vibration processing with the selected modes will be the most rational.

The developed procedure for designing vibration technological processes in the environment of free abrasive of particularly accurate parts has found practical application at machine-building enterprises of the parts production for agricultural machinery.

\section{References}

1. M. Tamarkin, E. Tishchenko, Basics for Optimization of Free Abrasive Machining Processes (Research monography) (LAP LAMBERT Academic Publishing, Saarboniken, Germany 2015)

2. V.A. Lebedev, V.V. Ivanov, V.P. Fedorov, IOP Conference Series: Materials Science and Engineering, 01216 (2016)

3. K. Hamouda, H. Bournine, M. Tamarkin, et al., Materials Science 52(2), 216-221 (2016) DOI: 10.1007/s11003-016-9946-9

4. V.V. Ivanov, V.A. Lebedev, I.A. Pinahin, Journal of Friction and Wear 35(4), 339-342 (2014) DOI: $10.3103 /$ S1068366614040059 
5. N.M. Antonova, V.S. Berezovsky, A.P. Babichev, Journal of Surface Investigation: XRay, Synchrotron and Neutron Techniques 11(5), 955-959 (2017) DOI: $10.1134 / \mathrm{S} 1027451017050032$

6. M. Tamarkin, E. Tishchenko, A. Shvedova, Russian Engineering Research 38(9), 726727 (2018) DOI: 10.3103/S1068798X18090277

7. B.S. Glazman, Y.V. Korol'kov, Y.V. Popov, Russian Engineering Research 34(1), 6064 (2014) DOI: 10.3103/S1068798X1401016X

8. M. Tamarkin, E. Tishchenko, A. Melnikov, E. Chernyshev, Advances in Intelligent Systems and Computing 983, 661-669 (2019) DOI: 10.1007/978-3-030-19868-8_64

9. M. Tamarkin, E. Tishchenko, I. Chukarina, T. Sosnitskaya, Proceedings of the VIII International Scientific Siberian Transport Forum - TransSiberia 20191 (2019)

10. K. Hamouda, T. Sayah, J.P. Ankudimov et al., Diffusion and Defect Data. Pt A Defect $\begin{array}{llll}\text { and Diffusion } & \text { Forum } & \text { 297-301, } & \text { 1103-1108 }\end{array}$ DOI: $10.4028 /$ www.scientific.net/DDF.297-301.110

11. G.A. Prokopetz, A.A. Azarova, A.A. Prokopetz, XIII International Scientific and Practical Conference "State and Prospects for the Development of Agribusiness INTERAGROMASH 2020" 175, 124-130 (2020)

12. Y.P. Ankudimov, I.V. Sadovaya, M.M. Chaava et al., Russian Engineering Research 38(9), 733-734 (2018) DOI: 10.3103/S1068798X18090058

13. M.A. Djema, K. Hamouda, T. Sayah et al., Diffusion and Defect Data. Pt A Defect and Diffusion Forum 326-328, 153-15 (2012)

14. M.A. Tamarkin, E.E. Tishchenko, O.A. Rozhnenko, Russian Engineering Research 33(5), 302-305 (2013) DOI: 10.3103/S1068798X1305016X

15. D. Saidi, M. Bouaziz, A.P. Babichev et al., Diffusion and Defect Data. Pt A Defect and Diffusion Forum 334-335, 7-12 (2013) DOI: 10.4028/www.scientific.net/DDF.334335.7 